\title{
Congenital Anterior Urethrocutaneous Fistula: Report of Three Cases
}

\author{
Akash Bihari Pati, Santosh Kumar Mahallik, Bikasha Bihary Tripathy, Manoj Kumar Mohanty
}

Department of Paediatric Surgery, All India Institute of Medical Sciences, Bhubaneswar

\section{ABSTRACT}

The Congenital anterior urethrocutaneous fistula is a rare entity with varied anatomic configurations. In this report, we present three different varieties of congenital anterior urethrocutaneous fistula. The anterior urethrocutaneous fistula was associated with no urethra, hypoplastic urethra, and normal urethra, distal to fistula, 1 in each patient. Each required a different treatment strategy. Postoperative outcome was good in all cases.

\section{Keywords: Congenital anterior urethrocutaneous fistula; Hypospadias; Urethroplasty}

Correspondence*: Akash Bihari Pati, Associate Professor, Paediatric Surgery, All India Institute of Medical Sciences, Sijua, Patrapada, Bhubaneswar, Odisha, India Pin-751014

E-mail: patiakash.ap@gmail.com

Submitted: 23-01-2018

Conflict of Interest: None
(C) 2018, Pati et al

Accepted: 23-03-2018

Source of Support: Nil

This is an open-access article distributed under the terms of the Creative Commons Attribution License, which permits unrestricted use, distribution, and reproduction in any medium provided the original work is properly cited.

\section{INTRODUCTION}

A urethrocutaneous fistula is usually acquired secondary to hypospadias repair. A congenital urethrocutaneous fistula is a rare entity and can occur at the anterior or posterior urethra. Congenital fistula from the anterior urethra is a rare entity and less than 50 cases have been reported in the literature.[1-3] We herein report three cases of congenital anterior urethrocutaneous fistula. Considerable controversy exists as to the etiology and management of this anomaly. The variation in anatomic configuration of the anomaly prompted us to report the cases.

\section{CASE SERIES}

Case 1: A 5-year-old uncircumcised male child presented with a passage of urine from an abnormal opening located at the ventral surface of the penis since birth. On examination, the glans was conical with the dorsal hooded prepuce. There was no meatus visible on the tip of the glans and a blind pit was present at the tip. The penis was curved ventrally with chordee. An oval opening of size approximately $8 \mathrm{~mm} \times 6 \mathrm{~mm}$ was present on the ventral aspect of the proximal penile shaft. A thin completely skin lined urethra was present between the opening and the glans of the penis. On probing the urethra distal to the opening a $6 \mathrm{Fr}$ infant feeding tube could be negotiated to the level of the corona (Figure 1). The urethra distal to the fistulous opening was hypoplastic and transparent. Feeding tube could be passed easily into the bladder through the proximal opening. Both the testes were descended and normal. The patient underwent two-stage urethroplasty. In first stage chordee correction with excision of the distal hypoplastic urethra was done. Second stage urethroplasty was done after 6 months. The patient is now passing a single straight stream of urine from the tip of the penis at 3-months follow up.

Case 2: A 4-year-old child presented with passing urine from an abnormal opening on the ventral surface of the penis. On examination, the prepuce was normal. Glans was conical with no meatus but a blind pit at the tip. 
The ventral part of the penis was completely skin covered. The meatus was located at the distal penile region and was about $8 \mathrm{~mm}$ wide. There was no chordee of the penile shaft. Both the testes were descended and normal in size. On catheterization, the feeding tube passed proximally into the bladder easily (Figure 2). A single stage Snodgrass urethroplasty was done with tubularization of the healthy skin at the site of the urethral plate with a dartos flap cover. Postoperatively, the patient recovered without any complications and is doing fine on the follow-up.

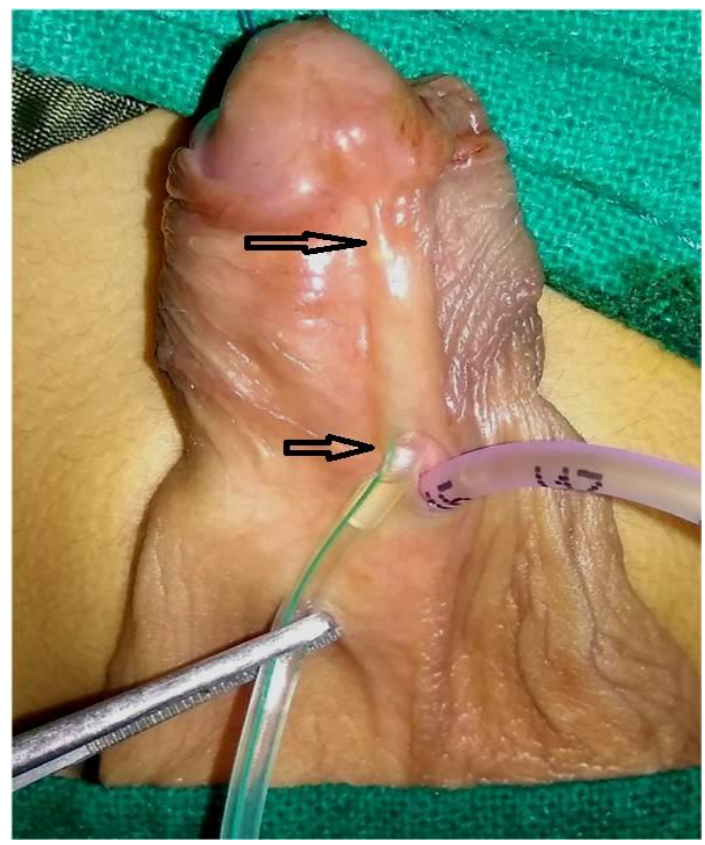

Figure 1: Catheter within the hypoplastic urethra (within arrows) distal to meatus up to the corona level.

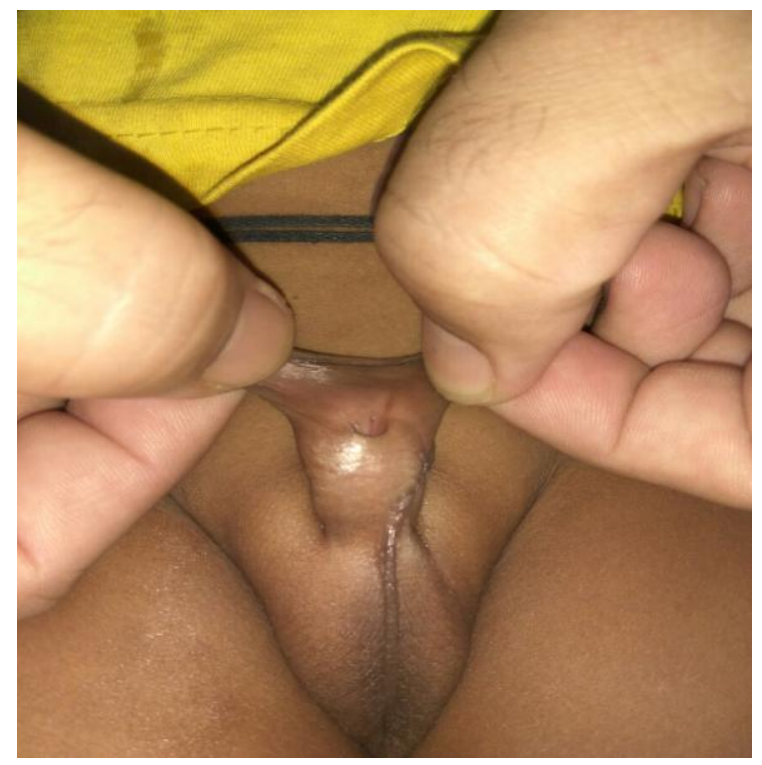

Figure 2: Distal penile hypospadias with healthy skin cover arrow mark distal to meatus.
Case 3: A 4-year-old child with anorectal malformation had a fistulous opening on the ventral surface of the mid shaft penis. The fistula was large with a diameter of $20 \mathrm{~mm}$. The distal part of the urethra beyond the fistula was of normal calibre and the meatus was opening at the tip of the penis. The defect was associated with chordee. Both the testes were normal and there was a perineal lipoma. At surgery, the chordee was corrected after degloving the penile skin. The fistula was closed primarily by 6-0 polydioxanone suture. A tunica vaginalis testis flap cover was placed over the suture line and skin closure done (Figure 3). The patient had an uneventful postoperative recovery and has no complications at 3 months follow up.

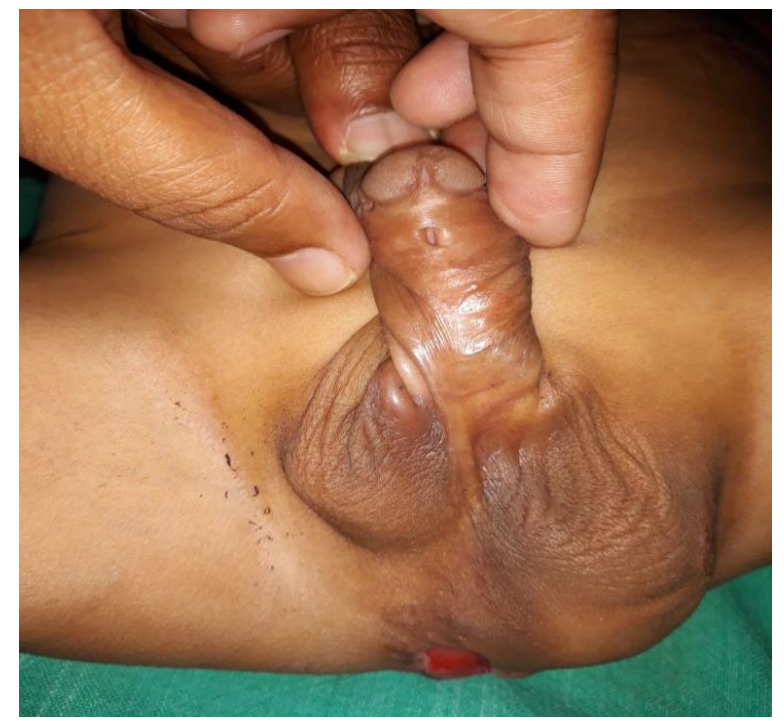

Figure 3: Normal urethra distal to fistula. Simple closure with tunica vaginalis flap arrow mark.

\section{DISCUSSION}

Congenital urethrocutaneous fistula in the anterior urethra is a rare anomaly. We presented three different variations of this anomaly i.e. having three different types of urethra distal to the fistulous opening. The urethra distal to the fistulous opening was hypoplastic in the first case, absent in the second case and normal in the third case. The management of the cases is also different depending on the anatomy and structures distal to the fistula.

An anterior urethrocutaneous fistula may be an isolated anomaly or associated with other anomalies like anorectal malformations, genitourinary anomalies etc. A review of 47 reported cases showed the fistula to be located at the distal shaft in 24 patients; at the mid shaft in 13 , at a penoscrotal junction in 3 ; and at an unspecified location in 7 cases.[1] The aetiology of congenital 
urethrocutaneous fistula is unknown. The most possible cause of this anomaly is a localized deficiency of a portion of the urethral plate. Under androgen stimulation, the genital tubercle elongates and the mesoderm in the urethral fold fuses in the midline enclosing the urethra. Goldstein postulated that there is a transient deficiency or inhibition of the local effect of the testosterone leading to the failed closure of the urethral groove.[2] This anomaly raises doubt about the postulation that the urethral folds fuse from proximal to distal penile regions serially, like a closing zipper.[3] Baskin et al. proposed the formation of a urethral seam at the edges of the urethral folds which fuses in the midline.[4]. A defect in the seam may explain for a focal defect in urethral fusion resulting in a congenital urethrocutaneous fistula. Congenital urethral fistula also postulated to have resulted from an embryonic urethral blowout resulting from a distal urethral obstruction.

The ectodermal cells from the tip of the glans penetrate inwards to form a cord which later canalizes. This canal joins with the penile urethra which has already formed at that time. The first case described above had a patent urethra from the fistula site up to the corona level though hypoplastic. This may be due to an associated defect in canalization of the glanular ectodermal urethra.

Two types of congenital anterior urethrocutaneous fistulae have been described. The first variety has an isolated urethrocutaneous fistula with intact distal urethra and spongiosum, normal foreskin and without any chordee or hypospadias. Our third case fell in this variety; however, he had skin level chordee which was corrected after degloving the penile skin. The other type is associated with hypospadias with chordee and dorsal hood with or without a distal spongiosal or urethral defect. The first and second cases described above belonged to this type.

Treatment of congenital urethrocutaneous fistulae is tailored depending upon the defect. According to Maarafie and Azmy, the urethra beyond the fistula dictates the type of surgery. If it is congenitally defective then simple closure is usually unsuccessful and the defective urethra should be opened and converted into a hypospadias.[5] In our series, the first case had hypoplastic urethra covered by a transparent membrane of skin distal to the urethrocutaneous fistula and the glanular urethra was deficient. Chordee correction was done by excising the thin hypoplastic urethra and staged urethroplasty done, whereas in the third case the distal part was healthy. We had performed a simple fistula closure along with a tunica vaginalis flap cover, which was successful in our second case; however, skin distal to meatal opening was tubularized.

In conclusion, congenital anterior urethrocutaneous fistula is a rare anomaly. The management of the condition depends upon the type of the defect distal to the urethral fistula. We could successfully manage the cases with excising the hypoplastic distal urethra in the first case, tubularizing the healthy skin in the second case, and simple closure of the fistula in the third case.

\section{AUTHOR'S CONTRIBUTIONS}

All authors contributed equally in concept, design, literature review, drafting manuscript, and approval of the final manuscript.

\section{CONSENT STATEMENT}

Authors declared that they have taken informed written consent, for publication of this report along with clinical photographs/material, from legal guardian of the patient with an understanding that every effort will be made to conceal the identity of the patient however it cannot be guaranteed.

\section{REFERENCES}

1. Cheng SY, Chen SJ, Lai HS. Congenital anterior urethrocutaneous fistula at the penoscrotal junction with proximal penile megalourethra: A case report. J Radiol Case Rep. 2016; 10:33-7.

2. Rashid KA, Kureel SN, Tandon RK. Congenital anterior penile isolated urethrocutaneous fistula: A case report. Afr J Paediatr Surg. 2008; 5:52-3.

3. Li Y, Sinclair A, Cao M, Shen J, Choudhry S, Botta S, et al. Canalization of the urethral plate precedes fusion of the urethral folds during male penile urethral development: the double zipper hypothesis. J Urol. 2015; 193:1353-9.

4. Baskin LS, Liu W, Bastacky J, et al. Anatomical Studies of the Mouse Genital Tubercle. In: Baskin LS, editor. Hypospadias and genital development, New York: Kluwer Academic/Plenum; 2004. p.103-121.

5. Maarafie A, Azmy AF. Congenital fistula of the penile urethra. Br J Urol. 1997; 79:814. 